\title{
RELAÇÃO K:Ca:Mg NA SOLUÇÃO NUTRITIVA PARA A PRODUÇÃO DE MINITOMATE CULTIVADO EM SUBSTRATO
}

\section{CAMILA ABRAHÃO' ${ }^{1}$; ROBERTO LYRA VILLAS BÔAS ${ }^{1}$ E LEONARDO THEODORO BULL ${ }^{1}$}

\footnotetext{
${ }^{1}$ Departamento de Solos e Recursos Ambientais, Faculdade de Ciências Agronômicas, Universidade Estadual Paulista "Julio de Mesquita Filho", Botucatu, SP. caabrahao99@gmail.com; rlvboas@ fca.unesp.br; bull@fca.unesp.br.
}

\section{RESUMO}

O estudo teve como objetivo avaliar seis relações $\mathrm{K}: \mathrm{Ca}: \mathrm{Mg}$ na produção de duas cultivares de minitomate cultivadas em substrato, sob ambiente protegido, em duas épocas de cultivo. $\mathrm{O}$ delineamento foi em blocos casualizados com quatro repetições e doze tratamentos, compostos por duas cultivares de minitomate (Sweet Million e Sweet Grape) e seis relações $\mathrm{K}: \mathrm{Ca}: \mathrm{Mg}$ (4:3:1, 6:3:1, 6:4,5:1, 2,7:3:1, 2,7:2:1, 4:2:1) nas soluções nutritivas. Nos dois experimentos observou-se que as soluções nutritivas que apresentaram as maiores concentrações de $\mathrm{Mg}$, $\left(75 \mathrm{mg} \mathrm{L}^{-1}\right)$, e as menores de $\mathrm{Ca}$, $\left(150 \mathrm{mg} \mathrm{L}^{-1}\right)$, corresponderam ao maior teor desse nutriente na massa seca das plantas. A cultivar Sweet Million apresentou maior produção $\left(1,69 \mathrm{~kg}_{\text {planta }}^{-1}\right.$ e 1,52 kg planta $\left.{ }^{-1}\right)$, maior número de frutos por planta $(227 \mathrm{e}$ 236) e maior eficiência no uso da água $\left(29,1 \mathrm{~kg} \mathrm{~m}^{-3}\right.$ e $\left.25,3 \mathrm{~kg} \mathrm{~m}^{-3}\right)$, porém a cultivar Sweet Grape apresentou frutos com maior peso médio $(9,0 \mathrm{~g}$ e $8,8 \mathrm{~g})$ e maior teor de macronutrientes nas folhas. Em ambos os ciclos de cultivo, as diferentes relações $\mathrm{K}: \mathrm{Ca}: \mathrm{Mg}$ influenciaram somente nos teores de macronutrientes das diferentes cultivares de minitomate cultivadas em substrato, não afetando a produção e a eficiência do uso da água. O primeiro ciclo de cultivo apresentou os maiores teores de N, K, Ca e S.

Palavras-chave: Solanum lycopersicum, fertirrigação, fibra de coco, nutrição de plantas, cultivo protegido

\section{ABRAHÃO, C.; VILLAS BÔAS, R.L.; BULL, L.T. K:CA:MG RATIO IN NUTRIENT SOLUTION FOR PRODUCTION OF MINI TOMATO GROWN IN SUBSTRATE}

\section{ABSTRACT}

The study was conducted to evaluate six $\mathrm{K}: \mathrm{Ca}: \mathrm{Mg}$ ratios for production of two cultivars of mini tomato grown in substrate, in a greenhouse, during two growing seasons. The experimental design was randomized blocks with four replications and twelve treatments using both cultivars of mini tomato (Sweet Million and Sweet Grape) and six K:Ca:Mg ratios $(4: 3: 1,6: 3: 1,6: 4.5: 1,2.7: 3: 1,2.7: 2: 1,4: 2: 1)$ in nutrient solutions. In both experiments, nutrient solutions with the highest concentrations of $\mathrm{Mg}$, (75 mg L-1) and the lowest concentrations of $\mathrm{Ca}$, (150 mg L-1) led to the highest concentrations of those nutrients in plant dry matter. The Sweet Million cultivar had higher yield (1.69 kg plant-1 and $1.52 \mathrm{~kg}$ 
plant-1), number of fruits per plant (227 and 236), and water use efficiency (29.1 kg m-3 and $25.3 \mathrm{~kg} \mathrm{~m}-3)$. However, the Sweet Grape cultivar had fruits of higher mean weight $(9.0 \mathrm{~g}$ and $8.8 \mathrm{~g}$ ) and macronutrient content in the leaves. In both crop cycles, the different $\mathrm{K}: \mathrm{Ca}: \mathrm{Mg}$ ratios affected only the macronutrient contents of the mini tomato plants grown in substrate, with no effect on yield and water use efficiency. The first crop cycle showed the highest N, K, Ca and S content.

Keywords: Solanum lycopersicum, fertigation, coconut fiber, plant nutrition, greenhouse.

\section{INTRODUÇÃO}

O tomateiro (Solanum lycopersicum) é uma das hortaliças mais importantes no Brasil e no mundo. Um dos principais fatores para a expansão da cultura é o crescimento do seu consumo. Segundo a FAOSTAT (FAO, 2013), no ano de 2011, a produção mundial de tomate foi por volta de 159 milhões de toneladas provenientes de aproximadamente 5 milhões de hectares. No Brasil em uma área de aproximadamente 71,5 mil hectares a produção foi de 4,4 milhões de toneladas.

Dentre as diversas cultivares de tomate, a demanda e a oferta pelo minitomate têm aumentado nos últimos anos, pois além de ser muito saboroso, é bastante atrativo, sendo muito utilizado na ornamentação de pratos e no preparo de saladas. Para os produtores, o interesse em cultivar esse grupo de tomate se deve ao alto valor agregado que apresenta no mercado.

Atualmente, as exigências do mercado por produtos de alta qualidade e diferenciados estimulam os produtores a buscarem por novas tecnologias e pelo aprimoramento das práticas de manejo do tomateiro. Dentre os sistemas de produção, o cultivo em substratos, quando comparado ao cultivo convencional, possibilita a obtenção de produtos de melhor qualidade devido entre outros fatores, a maior uniformidade na aplicação de água e de nutrientes (HAO et al., 2000; DUMAS et al., 2003), além de diminuir os impactos ambientais causados pela lixiviação destes (STANGHELLINI et al., 1998).

O cultivo de minitomate vem sendo realizado, principalmente, em substrato sob ambiente protegido, mas o sucesso do cultivo em substrato depende da otimização de diversos fatores, dentre os quais se destacam as práticas de irrigação e de nutrição das plantas (SOARES et al., 2005). Todos os nutrientes devem ser fornecidos em níveis compatíveis as exigências de cada espécie ou cultivar e de acordo com a sua fase de desenvolvimento (HAAG et al., 1993). Assim, as concentrações de nutrientes na solução são essenciais para elevar a produtividade e melhorar a qualidade do produto (FURLANI et al., 1999; DORAIS et al., 2001). Sabe-se que um nutriente pode afetar a absorção e a distribuição de outros elementos por competição, como ocorre entre os íons potássio $\left(\mathrm{K}^{+}\right)$, cálcio $\left(\mathrm{Ca}^{2+}\right)$ e magnésio $\left(\mathrm{Mg}^{2+}\right)$ (MARSCHENER, 1995). Os cátions monovalentes são absorvidos mais rapidamente pelas raízes do que os divalentes, assim o potássio compete fortemente na absorção de cálcio e de magnésio. Portanto, a relação adequada entre eles é fundamental para tornar máxima a absorção desses elementos, uma vez que o excesso de Ca também pode reduzir a absorção de K e de Mg (ASSIS, 1995; PAIVA et al., 1998).

O cultivo em substratos com a utilização da fertirrigação promove o aumento da produtividade e da qualidade dos frutos produzidos, pois possibilita às plantas a quantidade de nutrientes adequada para cada estádio de desenvolvimento da cultura (CHARLO et al., 2009).

No cultivo de minitomate há falta de informações sobre as relações entre potássio, 
cálcio e magnésio em solução nutritiva, principalmente no cultivo em substratos, na qual a carência de pesquisas é ainda maior. Portanto, o objetivo deste trabalho foi estudar os efeitos de seis relações $\mathrm{K}: \mathrm{Ca}: \mathrm{Mg}$ na solução nutritiva sobre a produção de duas cultivares de minitomate em substrato fibra de coco, sob ambiente protegido, em duas épocas de cultivo.

\section{MATERIAL E MÉTODOS}

Os experimentos foram conduzidos em ambiente protegido, sendo utilizado o delineamento experimental de blocos casualizados, com parcelas subdivididas, com quatro repetições e doze tratamentos, composto por relações $\mathrm{K}: \mathrm{Ca}: \mathrm{Mg}$ (R1=4:3:1; R2=6:3:1; $\mathrm{R} 3=6: 4,5: 1 ; \mathrm{R} 4=2,7: 3: 1 ; \mathrm{R} 5=2,7: 2: 1 ; \mathrm{R} 6=4: 2: 1)$ e duas cultivares de minitomates $(\mathrm{C} 1=$ Sweet Grape e C2=Sweet Million). As parcelas foram constituídas pelas diferentes soluções nutritivas e as subparcelas pelas cultivares. Cada parcela foi composta por seis plantas, sendo divididas em subparcelas, que foram compostas por três plantas de cada cultivar.

Os experimentos foram conduzidos durante os períodos de janeiro a junho e de julho a dezembro de 2010. As mudas do primeiro experimento foram produzidas em bandejas de poliestireno expandido de 200 células preenchidas com fibra de coco. Foram transplantadas no dia 13/01/2010 para vasos de oito litros preenchidos com substrato fibra de coco, no espaçamento de 0,30 metros entre plantas e 1,0 metro entre linhas. As mudas do segundo experimento foram produzidas nas mesmas condições do que o primeiro experimento, sendo transplantadas no dia 27/07/2010.

Em ambos os experimentos, todas as soluções nutritivas apresentaram as seguintes concentrações de $\mathrm{N}$, S e P: $180 \mathrm{mg} \mathrm{L}^{-1}, 85 \mathrm{mg} \mathrm{L}^{-1}$ e $50 \mathrm{mg} \mathrm{L}^{-1}$, respectivamente. As relações entre os cátions $\left(\mathrm{K}^{+}, \mathrm{Ca}^{++} \mathrm{e} \mathrm{Mg}^{++}\right)$constam na Tabela 1 .

Tabela 1. Relações K:Ca:Mg nas soluções nutritivas para o desenvolvimento do tomateiro.

\begin{tabular}{lcccccc}
\hline Nutrientes & \multicolumn{6}{c}{ Concentração $\left(\mathbf{m g ~ L}^{-1}\right)$} \\
\hline & $\mathbf{4 : 3 : 1}$ & $\mathbf{6 : 3 : 1}$ & $\mathbf{6 : 4 , 5 : 1}$ & $\mathbf{2 , 7 : 3 : 1}$ & $\mathbf{2 , 7 : 2 : 1}$ & $\mathbf{4 : 2 : 1}$ \\
$\mathbf{K}$ & 200 & 300 & 300 & 200 & 200 & 300 \\
$\mathbf{C a}$ & 150 & 150 & 225 & 225 & 150 & 150 \\
$\mathbf{M g}$ & 50 & 50 & 50 & 75 & 75 & 75 \\
\hline
\end{tabular}

Em todos os tratamentos as concentrações de micronutrientes foram: $1,48 \mathrm{mg} \mathrm{L}^{-1}$ de Fe; $0,37 \mathrm{mg} \mathrm{L}^{-1}$ de $\mathrm{Mn} ; 0,37 \mathrm{mg} \mathrm{L}^{-1}$ de $\mathrm{Cu} ; 0,15 \mathrm{mg} \mathrm{L}^{-1}$ de $\mathrm{Zn} ; 0,37 \mathrm{mg} \mathrm{L}^{-1}$ de B e $0,07 \mathrm{mg} \mathrm{L}^{-1}$ de Mo. As soluções nutritivas foram preparadas a partir dos seguintes fertilizantes: nitrato de cálcio $(20 \% \mathrm{Ca} ; 15 \% \mathrm{~N})$; nitrato de potássio $(36,5 \% \mathrm{~K} ; 13 \% \mathrm{~N})$; nitrato de amônio $(33 \% \mathrm{~N})$; nitrato de magnésio $(9 \% \mathrm{Mg} ; 11 \% \mathrm{~N})$; fosfato monopotássico $(22,3 \% \mathrm{P} ; 27,4 \% \mathrm{~K})$; sulfato de magnésio $(9,5 \% \mathrm{Mg} ; 12 \% \mathrm{~S})$; sulfato de potássio $(41,5 \% \mathrm{~K} ; 17 \% \mathrm{~S})$; cloreto de potássio $(36,5 \% \mathrm{~K})$; cloreto de cálcio $(27 \% \mathrm{Ca})$; e o produto comercial denominado Conmicros Standard que apresenta as garantias em porcentagem dos seguintes nutrientes $(\mathrm{B}=1,82 \%$; $\mathrm{Cu}-$ EDTA $=1,82 \% ;$ Fe-EDTA=7,26\%; Mo=0,36\%; Mn-EDTA $=1,82 \% ;$ Zn-EDTA $=0,73 \%$ e $\mathrm{Ni}=0,36 \%)$.

Para os dois experimentos o controle da irrigação foi calculado pela perda de água das plantas, sendo mensurada pelo peso de um vaso por tratamento em cada repetição, por meio de balança digital. Para isso, foi alcançada a capacidade de campo dos vasos, e posteriormente realizada somente a reposição da perda diária de água. Tanto a aplicação da água quanto a aplicação da solução nutritiva foram realizadas por meio do mesmo sistema de irrigação. A aplicação da solução nutritiva foi realizada diariamente, sendo dividida em dois períodos do 
dia (manhã e final da tarde). Após cada aplicação de solução nutritiva o sistema era lavado 1 minuto com água, para evitar a mistura das soluções nutritivas no sistema de irrigação, bem como o entupimento dos gotejadores e o resíduo de fertilizantes nas mangueiras. No primeiro ciclo de cultivo, foram aplicados $58 \mathrm{~L}$ de água+solução nutritiva para cada planta, e para o segundo ciclo de cultivo aproximadamente $61 \mathrm{~L}$ de água+solução nutritiva para cada planta. Para o primeiro experimento foi usado um conjunto moto-bomba de $0,5 \mathrm{CV}$, que alimentava as linhas de irrigação (um tratamento por vez), realizada por gotejadores autocompensantes, com vazão nominal de $2 \mathrm{~L} \mathrm{~h}^{-1}$. Para o segundo experimento foi usado três conjuntos motobomba de 0,25 CV, que alimentavam as linhas de irrigação (três tratamentos por vez). Os fertilizantes foram aplicados através da solução nutritiva que era preparada duas vezes por semana em reservatórios de $90 \mathrm{~L}$, e aplicadas separadamente para cada tratamento, sendo controladas através de registros. Os reservatórios e a água de irrigação foram abastecidos por uma caixa d'água de 1000L.

Nos dois experimentos o manejo, a condução e o tutoramento das plantas ocorreram de formas iguais, sendo que as plantas de tomate foram tutoradas em haste única, no sistema vertical com fitilhos amarrados em arames, que foram colocados na horizontal presos em barras de metalon na parte superior de mourões de 2,50 $\mathrm{m}$ de altura. A retirada dos brotos foi realizada com o auxílio de uma tesoura de poda, quando o broto atingisse de 5 a $10 \mathrm{~cm}$, e o tutoramento foi realizado sempre que necessário. O broto apical das plantas foi eliminado acima do sexto cacho floral, deixando uma folha acima deste.

No primeiro ciclo a colheita iniciou-se aos 60 dias após o transplante (DAT) e no segundo ciclo aos 70 DAT, sendo realizada duas vezes por semana, os quais foram colhidos somente os frutos maduros. Em ambos os experimentos, foram avaliados a produção, número de frutos por planta, massa média de frutos por planta, teor de nutrientes nas folhas e eficiência do uso da água. Para a determinação da produção e massa média de frutos por planta, os frutos maduros foram pesados por meio de balança analítica. A quantificação do número de frutos por planta foi realizado por meio da contagem total dos frutos colhidos sem defeitos. Para o teor de nutrientes nas folhas, em ambos os ciclos de cultivo, no período que compreendeu ao florescimento e ao aparecimento dos primeiros frutos maduros, a quarta folha a partir do ápice (folha diagnose) foi coletada, num total de três folhas por tratamento. Foram lavadas, secadas em estufa à $65^{\circ} \mathrm{C}$ e moídas em moinho tipo Wiley, para as avaliações dos teores de macronutrientes, $\mathrm{B}, \mathrm{Cu}, \mathrm{Fe}, \mathrm{Mn}$ e $\mathrm{Zn}$, segundo metodologia descrita por MALAVOLTA et al. (1997). A eficiência do uso da água $\left(\mathrm{kg} \mathrm{m}^{-3}\right)$, para os dois experimentos, foi calculada por meio da relação entre a produção total do tomate $\left(\mathrm{kg} \mathrm{planta}^{-1}\right)$ e a quantidade total de água aplicada durante o ciclo de cultivo $(\mathrm{mL})$.

Os resultados dos experimentos foram submetidos à análise de variância e as médias comparadas pelo teste de Tukey com $\mathrm{p}>0,05$ e análise conjunta por meio do procedimento PROC GLM do programa estatístico SAS (Statistical Analysis Software).

\section{RESULTADOS E DISCUSSÃO}

Os valores médios da produção em kg por planta, número de frutos por planta e massa média dos frutos nas diferentes relações de $\mathrm{K}: \mathrm{Ca}: \mathrm{Mg}$ e nas duas cultivares de minitomate dos experimentos, estão apresentados na Tabela 2. 
Tabela 2. Valores médios de produção $\left(\mathrm{kg}_{\text {planta }}{ }^{-1}\right)$, número de frutos por planta e peso médio dos frutos $(\mathrm{g})$ em minitomate, em função das relações $\mathrm{K}: \mathrm{Ca}: \mathrm{Mg}$ e das cultivares.

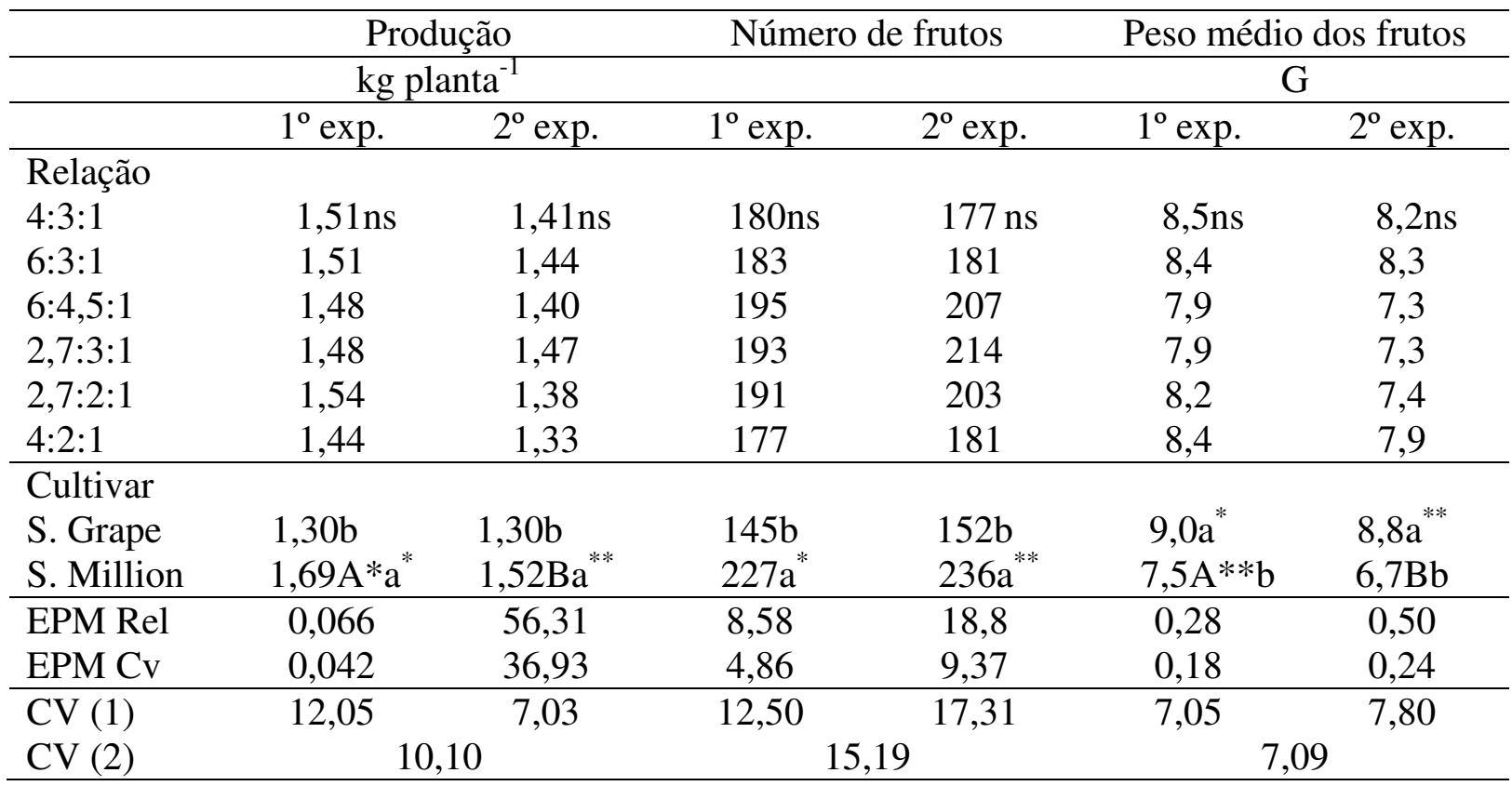

Rel: relação; $\mathrm{Cv}$ : cultivar. EPM: erro padrão da média; $\mathrm{CV}(1)$ : coeficiente de variação em cada experimento; $\mathrm{CV}(2)$ : coeficiente de variação entre experimentos. Médias seguidas de mesma letra maiúscula ou sem letra na linha não diferem entre si pelo Teste F. Médias seguidas de mesma letra minúscula ou sem letra na coluna não diferem entre si pelo Teste de Tukey, a 5\% de probabilidade. ns: não significativo ao nível de 5\% de probabilidade. **, *: significativo a 1 e $5 \%$ de probabilidade, respectivamente.

Verificou-se através da análise estatística que não houve interação entre as diferentes relações $\mathrm{K}: \mathrm{Ca}: \mathrm{Mg}$ e as cultivares. Isso pode ter ocorrido devido à adaptabilidade que foi observada nas cultivares avaliadas em se desenvolver sob diferentes concentrações desses nutrientes.

Holcman (2009) estudando duas relações K:N (2:1 e 3:1) em minitomates, Sweet Grape e Sweet Million, também não observou diferenças estatísticas para produção e massa média de frutos. No entanto, observou que a relação 3:1 proporcionou maior quantidade no número de frutos maiores (61,5\% S. Grape e $69,5 \% \mathrm{~S}$. Million), enquanto que a relação 2:1 maior número de frutos menores (42,5\% S. Grape e 33\% S. Million).

Fanasca et al. (2006) avaliando três relações K:Ca:Mg na solução nutritiva para duas cultivares de tomate, observaram efeito significativo sobre a produção total, comercial e número de frutos de tomate por planta, sendo que a solução nutritiva que continha maior concentração de $\mathrm{Ca}\left(284 \mathrm{mg} \mathrm{L}^{-1}\right)$, proporcionou os maiores valores observados para estas variáveis $\left(6,2 \mathrm{~kg}_{\text {planta }}{ }^{-1}, 5,6 \mathrm{~kg}_{\text {planta }}{ }^{-1}\right.$ e 46,4 frutos por planta, respectivamente), pois reduziu a porcentagem de frutos com podridão apical.

Bull et al. (1998) observaram que os menores valores da relação $(\mathrm{Ca}+\mathrm{Mg}) / \mathrm{K}, 3,6 \mathrm{e}$ 1,9, no solo inibiram o desenvolvimento das plantas e a produção dos bulbos de alho vernalizado, devido ao desbalanço catiônico entre $\mathrm{Ca}$ e $\mathrm{Mg}$. Esses mesmos autores verificaram tendência de aumento na produção de bulbos com a dose de potássio aplicado até que se atinja valores de relação $(\mathrm{Ca}+\mathrm{Mg}) / \mathrm{K}$ entre 18,5 e 26,3.

Lima et al. (1981) observaram que as produções máximas de matéria seca em tomate cv. Kada ocorreram entre relações de 10,5 a 26,3. 
Através da análise de variância observou-se efeito significativo para as cultivares sobre produção, número de frutos por planta e peso médio dos frutos em ambos os experimentos. A produção do minitomate Sweet Million foi $23 \%$ maior do que a variedade Sweet Grape para o primeiro experimento e para o segundo apresentou um aumento de $14,5 \%$. Essa maior produtividade pode ter ocorrido devido ao maior número de frutos produzidos pela cultivar Sweet Million em relação à variedade Sweet Grape. Verifica-se também que pela análise conjunta houve efeito significativo entre as duas épocas de cultivo para produção em relação a cultivar Sweet Million que apresentou produção maior de $10 \%$ no primeiro experimento em relação ao segundo experimento.

Holcman (2009) avaliando as mesmas cultivares Sweet Grape e Sweet Million também obteve maior produção da cultivar Sweet Million em relação à Sweet Grape (12\%).

Em relação ao peso médio dos frutos a cultivar Sweet Grape obteve média superior à cultivar Sweet Million, devido ao menor número de frutos produzidos por essa cultivar, obtendo dessa forma frutos maiores. Enquanto que a cultivar Sweet Million produziu maior quantidade de frutos, ocorrendo maior competição entre os frutos na planta, produzindo frutos de menor peso. Observou-se que houve diferença significativa para a análise conjunta dos dados para a cultivar Sweet Million, no qual o peso médio dos frutos do primeiro ciclo de cultivo foi 10,67\% superior em relação ao segundo ciclo. Holcman (2009) observou que a cultivar Sweet Million obteve peso médio de frutos superior $(9,3 \mathrm{~g})$ aos da cultivar Sweet Grape $(8,6 \mathrm{~g})$, pois produziu quantidade menor de frutos ao longo do ciclo, gerando frutos de peso maior, apresentando superioridade de 7,5\%.

Para os teores de nutrientes nas folhas pode se observar através da análise de variância que houve efeito das diferentes relações $\mathrm{K}: \mathrm{Ca}: \mathrm{Mg}$ apenas sobre os teores dos nutrientes $\mathrm{Mg}$ e $\mathrm{S}(\mathrm{p}<0,05)$, conforme apresentado na Tabelas 3 e 4.

Tabela 3. Teores de $\mathrm{N}, \mathrm{P}$ e $\mathrm{K}\left(\mathrm{g} \mathrm{kg}^{-1}\right)$ em folhas de mini tomate, analisados aos $60 \mathrm{DAT}$, em função das relações $\mathrm{K}: \mathrm{Ca}: \mathrm{Mg}$ e das cultivares.

\begin{tabular}{|c|c|c|c|c|c|c|}
\hline & \multicolumn{2}{|c|}{$\mathrm{N}$} & \multicolumn{2}{|c|}{$\mathrm{P}$} & \multicolumn{2}{|c|}{ K } \\
\hline & \multicolumn{6}{|c|}{$\mathrm{g} \mathrm{kg}^{-1}$} \\
\hline & $1^{\circ}$ exp. & $2^{\circ}$ exp. & $1^{\circ}$ exp. & $2^{\circ} \exp$ & $1^{\circ}$ exp. & $2^{\circ}$ exp. \\
\hline \multicolumn{7}{|l|}{ Relação } \\
\hline $4: 3: 1$ & $39 A^{*}$ & $34 B$ & $6,1 \mathrm{~ns}$ & $5,6 \mathrm{~ns}$ & 28 & 27 \\
\hline $6: 3: 1$ & $40 A^{*}$ & $37 \mathrm{~B}$ & 6,3 & 5,7 & 31 & 29 \\
\hline $6: 4,5: 1$ & $40 \mathrm{~A} * *$ & $34 B$ & 5,9 & 5,5 & $31 A^{*}$ & $26 B$ \\
\hline $2,7: 3: 1$ & $40 A^{*}$ & $36 \mathrm{~B}$ & 6,1 & 5,6 & $31 A^{*}$ & $27 \mathrm{~B}$ \\
\hline $2,7: 2: 1$ & $39 A^{*}$ & $35 B$ & 6,1 & 5,6 & $32 \mathrm{~A} *$ & $25 \mathrm{~B}$ \\
\hline $4: 2: 1$ & 37 & 36 & 6,0 & 5,9 & $31 A^{*}$ & $27 \mathrm{~B}$ \\
\hline \multicolumn{7}{|l|}{ Cultivar } \\
\hline S.Grape & $41 \mathrm{~A} * \mathrm{a} *$ & $38 \mathrm{Ba} * *$ & $6,3 a *$ & $6,3 a^{* *}$ & $32 \mathrm{~A} * \mathrm{a} *$ & $29 \mathrm{Ba} * *$ \\
\hline S.Million & $38 A^{* *} \mathrm{~b}$ & $33 \mathrm{Bb}$ & $5,8 \mathrm{~A} * * \mathrm{~b}$ & $5,0 \mathrm{Bb}$ & $30 A^{* * b}$ & $25 \mathrm{Bb}$ \\
\hline EPM Rel & 0,93 & 1,01 & 0,19 & 0,13 & 0,89 & 1,21 \\
\hline EPM Cv & 0,65 & 0,67 & 0,1 & 0,08 & 0,51 & 0,87 \\
\hline CV (1) & 5,87 & 7,63 & 6,52 & 6,47 & 9,60 & 11,80 \\
\hline CV (2) & \multicolumn{2}{|c|}{6,48} & \multicolumn{2}{|c|}{6,40} & \multicolumn{2}{|c|}{10,09} \\
\hline
\end{tabular}


Tabela 4. Teores de $\mathrm{Ca}, \mathrm{Mg}$ e $\mathrm{S}\left(\mathrm{g} \mathrm{kg}^{-1}\right)$ em folhas de mini tomate, analisados aos $60 \mathrm{DAT}$, em função das relações K:Ca:Mg e das cultivares.

\begin{tabular}{|c|c|c|c|c|c|c|}
\hline & \multicolumn{2}{|c|}{$\mathrm{Ca}$} & \multicolumn{2}{|c|}{$\mathrm{Mg}$} & \multicolumn{2}{|c|}{ S } \\
\hline \multicolumn{7}{|c|}{$\mathrm{g} \mathrm{kg}^{-1}$} \\
\hline & $1^{\circ}$ exp. & $2^{\circ} \exp$ & $1^{\circ}$ exp. & $2^{\circ} \exp$ & $1^{\circ}$ exp. & $2^{\circ}$ exp. \\
\hline \multicolumn{7}{|l|}{ Relação } \\
\hline 4:3:1 & $11 A^{* *}$ & $6 \mathrm{Bc}$ & $5,6 a b$ & $5 \mathrm{~b}$ & $4,6 \mathrm{~A} * \mathrm{a}$ & $5,4 \mathrm{~B}$ \\
\hline 6:3:1 & $9 A^{*}$ & $6 \mathrm{Bc}$ & $5,2 b$ & $5 \mathrm{~b}$ & $4,1 \mathrm{~A} * \mathrm{a} *$ & $4,7 \mathrm{~B}$ \\
\hline $6: 4,5: 1$ & $11 \mathrm{~A} * *$ & $7 \mathrm{Bb}$ & $5,1 b$ & $4 \mathrm{c}$ & $3,5 \mathrm{~A} * * \mathrm{~b}$ & $4,9 \mathrm{~B}$ \\
\hline $2,7: 3: 1$ & $12 \mathrm{~A} *$ & $8 \mathrm{Ba}^{*}$ & $5,2 b$ & $5 \mathrm{~b}$ & $3,4 \mathrm{~A} * * \mathrm{~b}$ & $5,2 \mathrm{~B}$ \\
\hline $2,7: 2: 1$ & $9 A^{*}$ & $5 \mathrm{Bd}$ & $6,0 a^{*}$ & $6 a^{* *}$ & $3,6 \mathrm{~A} * \mathrm{~b}$ & $4,8 \mathrm{~B}$ \\
\hline $4: 2: 1$ & $9 A^{*}$ & $6 \mathrm{Bc}$ & $6,1 a^{*}$ & $6 a^{* *}$ & $3,6 A^{*} \mathrm{~b}$ & $4,7 \mathrm{~B}$ \\
\hline \multicolumn{7}{|l|}{ Cultivar } \\
\hline S.Grape & $9 \mathrm{~A} * * \mathrm{~b}$ & $6 \mathrm{Bb}$ & $5,1 b$ & 5,2 & $3,5 \mathrm{~A} * * \mathrm{~b}$ & $5,1 \mathrm{Ba}^{*}$ \\
\hline S.Million & $11 \mathrm{~A} * * \mathrm{a} *$ & $7 \mathrm{Ba}^{* *}$ & $6,0 \mathrm{~A} * * \mathrm{a} *$ & $5,2 \mathrm{~B}$ & $4,1 \mathrm{~A} * * \mathrm{a} * *$ & $4,8 \mathrm{Bb}$ \\
\hline EPM Rel & 0,90 & 0,28 & 0,20 & 0,11 & 0,16 & 0,23 \\
\hline EPM Cv & 0,45 & 0,15 & 0,10 & 0,06 & 0,09 & 0,13 \\
\hline CV (1) & 17,72 & 11,86 & 6,61 & 5,97 & 8,97 & 12 \\
\hline CV (2) & \multicolumn{2}{|c|}{16,41} & \multicolumn{2}{|c|}{6,41} & \multicolumn{2}{|c|}{10,17} \\
\hline
\end{tabular}

Rel: relação; Cv: cultivar. EPM: erro padrão da média; CV(1): coeficiente de variação em cada experimento; CV(2): coeficiente de variação entre experimentos. Médias seguidas de mesma letra maiúscula na linha não diferem entre si pelo Teste F. Médias seguidas de mesma letra minúscula na coluna não diferem entre si pelo Teste de Tukey, a 5\% de probabilidade. ns: não significativo ao nível de 5\% de probabilidade. **, *: significativo a 1 e $5 \%$ de probabilidade, respectivamente.

No primeiro ciclo de cultivo as soluções nutritivas que apresentaram maior concentração de $\mathrm{Mg}$, $\left(75 \mathrm{mg} \mathrm{L}^{-1}\right),(2,7: 2: 1)$ e $(4: 2: 1)$ corresponderam ao maior teor desse nutriente na massa seca das plantas, sendo os valores médios 6,0 e $6,1 \mathrm{~g} \mathrm{~kg}^{-1}$, respectivamente. Esse fato pode indicar que os teores nas folhas estão diretamente relacionados às concentrações dos nutrientes nas soluções utilizadas. Entretanto, a relação 2,7:3:1, que também continha maior concentração de $\mathrm{Mg}$, não mostrou efeito significativo, provavelmente pela diminuição da absorção do Mg causado por uma maior concentração de Ca nesta solução, e portanto, menor teor nas folhas das plantas. Os maiores teores de $\mathrm{S}, 4,6 \mathrm{~g}$ $\mathrm{kg}^{-1}$ e $4,1 \mathrm{~g} \mathrm{~kg}^{-1}$, foram observados nas relações 4:3:1 e 6:3:1, respectivamente, sendo que nas outras relações os valores médios não diferiram estatisticamente.

Em relação aos teores de macronutrientes nas folhas para o segundo experimento, observou-se por meio da análise de variância que houve efeito das diferentes relações $\mathrm{K}: \mathrm{Ca}: \mathrm{Mg}$ apenas sobre os teores dos nutrientes $\mathrm{Ca}$ e $\mathrm{Mg}$. Os maiores teores médios de $\mathrm{Ca}$ (8 e $7 \mathrm{mg} \mathrm{kg}^{-1}$ ) foram observados nas relações que continham as maiores concentrações de $\mathrm{Ca}$, sendo que o maior valor encontrado foi na solução nutritiva que apresentava menor relação $\mathrm{K}: \mathrm{Ca}$. Dependendo da concentração de K na solução nutritiva, pode haver uma diminuição da absorção de Ca pelas plantas, com isso essa menor relação não afetou a absorção de Ca pelo tomateiro. 
Em relação ao nutriente $\mathrm{Mg}$, o maior teor médio encontrado foi $6 \mathrm{mg} \mathrm{kg}^{-1}$, sendo observado nas relações que continham as maiores concentrações $\left(75 \mathrm{mg} \mathrm{L}^{-1}\right)$ de $\mathrm{Mg}$ e as menores de $\mathrm{Ca}\left(150 \mathrm{mg} \mathrm{L}^{-1}\right)$. Altas concentrações de Ca podem inibir ou diminuir a taxa de absorção de $\mathrm{Mg}$ pelas raízes das plantas.

Para as cultivares houve efeito significativo sobre as concentrações de todos os macronutrientes em ambos os ciclos de cultivo, com exceção do Mg no segundo ciclo. A cultivar Sweet Grape apresentou maiores concentrações na massa seca das folhas para os nutrientes N, P e K, entretanto para a cultivar Sweet Million, a maior concentração de nutrientes ocorreu para $\mathrm{Ca}, \mathrm{Mg}$ e $\mathrm{S}$ no primeiro ciclo de cultivo, conforme apresentado na Tabela 3.

Observou-se pela análise conjunta que houve diferença significativa entre os dois ciclos estudados para as diferentes relações $\mathrm{K}: \mathrm{Ca}: \mathrm{Mg}$ e para as cultivares. As concentrações de N, K, Ca e S na massa seca das folhas de minitomate foram maiores no primeiro ciclo de cultivo em relação ao segundo, assim como para as cultivares Sweet Grape e Sweet Million.

Em relação à eficiência do uso da água em $\mathrm{kg} \mathrm{m}^{-3}$, não foi observado efeito significativo de interação entre relações e cultivar, evidenciando que as diferentes soluções nutritivas aplicadas não influenciaram na eficiência do uso da água, conforme apresentado na Tabela 5 .

Tabela 5. Valores médios da eficiência do uso da água (EUA) em plantas de minitomate, em função das relações $\mathrm{K}: \mathrm{Ca}: \mathrm{Mg}$ e das cultivares.

\begin{tabular}{lcc}
\hline \multicolumn{3}{c}{ EUA } \\
$\mathrm{kg} \mathrm{m}^{-3}$ \\
\hline Relação & $1^{\circ}$ Experimento & $2^{\circ}$ Experimento \\
$4: 3: 1$ & $26,0 \mathrm{~ns}$ & $23,5 \mathrm{~ns}$ \\
$6: 3: 1$ & 26,0 & 24,0 \\
$6: 4,5: 1$ & 25,6 & 23,4 \\
$2,7: 3: 1$ & 25,5 & 24,6 \\
$2,7: 2: 1$ & 26,7 & 23,0 \\
$4: 2: 1$ & 24,8 & 22,3 \\
\hline Cultivar & & \\
S. Grape & $22,3 \mathrm{Ab}$ & $21,6 \mathrm{Bb}^{* *}$ \\
S. Million & $29,1 \mathrm{~A}{ }^{*} \mathrm{a}^{* *}$ & $25,3 \mathrm{~B}^{*} \mathrm{a}^{*}$ \\
\hline EPM Rel & 0,64 & 0,94 \\
EPM Cv & 0,37 & 0,62 \\
\hline CV (1) & 12,03 & 7,33 \\
CV (2) & 10,18 & 10,18 \\
\hline
\end{tabular}

Rel: relação; Cv: cultivar. EPM: erro padrão da média; $\mathrm{CV}(1)$ : coeficiente de variação em cada experimento; $\mathrm{CV}(2)$ : coeficiente de variação entre experimentos. Médias seguidas de mesma letra maiúscula na linha não diferem entre si pelo Teste F. Médias seguidas de mesma letra minúscula na coluna não diferem entre si pelo Teste de Tukey, a 5\% de probabilidade. ns: não significativo ao nível de 5\% de probabilidade. **, *: significativo a 1 e $5 \%$ de probabilidade, respectivamente.

O K é responsável pela mudança do turgor das células guarda durante o movimento estomático, sendo que com o aumento da concentração de $\mathrm{K}$ nas células guarda ocorre a absorção de água através das células adjacentes, ocasionando o aumento do turgor das células guarda e consequentemente a abertura dos estômatos (MARSCHNER, 1995). Segundo Mengel (2007), plantas insuficientemente supridas com K, podem perder o turgor quando 
expostas ao estresse hídrico. No presente estudo, apesar das diferentes relações $\mathrm{K}: \mathrm{Ca}: \mathrm{Mg}$ estes efeitos não foram observados.

No entanto, observa-se diferença estatística entre as diferentes cultivares. No primeiro e segundo experimento a cultivar Sweet Million apresentou maior eficiência do uso da água $\left(29,1 \mathrm{~kg} \mathrm{~m}^{-3}\right.$ e $25,3 \mathrm{~kg} \mathrm{~m}^{-3}$, respectivamente) em relação a cultivar Sweet Grape $\left(22,3 \mathrm{~kg} \mathrm{~m}^{-3} \mathrm{e}\right.$ $21,6 \mathrm{~kg} \mathrm{~m}^{-3}$, respectivamente), devido à menor produção de frutos desta. Não foram encontrados dados na literatura que mostrem valores médios ideais para as cultivares avaliadas no presente estudo. No entanto, o limite considerado ideal pela FAO (2001) para tomate varia entre 10 e $12 \mathrm{~kg} \mathrm{~m}^{-3}$. Soares e Faria (1983) observaram valor médio de $10,5 \mathrm{~kg}$ $\mathrm{m}^{-3}$ em tomate para uso industrial. Kalungu (2008) reportou valor médio de $13,4 \mathrm{~kg} \mathrm{~m}^{-3} \mathrm{em}$ tomate tipo longa vida variedade Débora Plus. Observa-se que os valores médios da eficiência do uso da água encontrados para as cultivares Sweet Grape e Sweet Million são superiores aos valores observados para outros tipos de tomate, mostrando que possivelmente os minitomates podem apresentar maior eficiência do uso da água ou ainda que o cultivo em substrato promoveu maior eficiência do uso da água com menores perdas e portanto, melhor aproveitamento. Segundo a análise conjunta dos dados, não houve diferença significativa para as diferentes relações de $\mathrm{K}, \mathrm{Ca}$ e $\mathrm{Mg}$, somente para as cultivares. Observou-se que as cultivares Sweet Grape e Sweet Million apresentaram valores maiores no primeiro ciclo de cultivo em relação ao segundo.

\section{CONCLUSÕES}

As diferentes relações de $\mathrm{K}: \mathrm{Ca}: \mathrm{Mg}$ não influenciam na produção e na eficiência do uso da água para minitomate. No entanto, afetam nos teores de nutrientes nas folhas indicando que os teores nas folhas estão diretamente relacionados às concentrações dos nutrientes nas soluções utilizadas.

As cultivares diferem entre sim quanto a produção, teor de nutriente nas folhas e na eficiência do uso da água, sendo que a cultivar Sweet Million apresenta maior produção e eficiência do uso da água em relação a cultivar Sweet Grape. Enquanto a cultivar Sweet Grape apresenta maior concentração dos macronutrientes em relação a cultivar Sweet Million.

As diferentes épocas de cultivo diferem entre si em relação as cultivares, sendo que o primeiro ciclo de cultivo apresentou os maiores valores quanto à produção, peso médio de frutos, teores de nutrientes e eficiência do uso da água, podendo ser considerado a época ideal para o cultivo do minitomate.

\section{REFERÊNCIAS BIBLIOGRÁFICAS}

ASSIS, R. P. de. Nutrição mineral e crescimento de mudas de dendezeiro (Elaeis guinensis Jacq.) em função de diferentes relações entre K, Ca e Mg na solução nutritiva. 1995. 41 f. Dissertação (Mestrado Agronomia)-Universidade Federal de Lavras, Lavras, 1995.

BÜLL, L.T.; VILLAS BÔAS, R.L.; NAKAGAWA, J. Variações no balanço catiônico do solo induzidas pela adubação potássica e efeitos na cultura do alho vernalizado. Scientia Agricola, Piracicaba, v.55, p.456-464, 1998. 
CHARLO, H. C. de O.; CASTOLDI, R.; FERNANDES, C.; VARGAS, P.F.; BRAZ, L.T. Cultivo de híbridos de pimentão amarelo em fibra da casca de coco. Horticultura Brasileira, Brasília, DF, v. 27, n. 2, p. 155-159, abr./jun. 2009.

DORAIS, M.; PAPADOPOULOS, A. P.; GOSSELIN, A. Influence of electric conductivity management on greenhouse tomato yield and fruit quality. Agronomie, Paris, v. 21, n. 4, p. 367-383, May/June 2001.

DUMAS, Y.; DADOMO, M.; DI LUCCA, G.; GROLIER, P. Effects of environmental factors and agricultural techniques on antioxidant content of tomatoes. Journal Science Food Agriculture, London, v. 83, n. 5, p. 369-382, Apr. 2003.

FANASCA, S.; COLLA, G.; MAIANI, G.; VENNERIA, E.; ROUPHAEL, Y.; AZZINI, E.; SACCARDO, F. Changes in antioxidant content of tomato fruits in response to cultivar and nutrient solution composition. Journal of Agriculture and Food Chemistry, Easton, Pa., US: American Chemical Society, v. 54, p. 4319-4325.

FAO. FAOSTAT 2013. Disponível em: <http://faostat.fao.org>. Acesso em: 10 jun. 2013.

FAO. New frontiers in capacity building in drainage. In: RITZEMA, H. P.; WOLTERS, W. WORKSHOP CAPACITY BUILDING FOR DRAINAGE IN NORTH AFRICA, 2001, Cairo. Proceedings... Rome: FAO, 2001. 1 CD-ROM.

FURLANI, P. R.; SILVEIRA, L.C.P.; BOLONHEZI, D.; FAQUIN, V. Cultivo hidropônico de plantas. Campinas: IAC, 1999. $50 \mathrm{p}$.

HAAG, H. P.; DECHEN, A.R.; CARMELLO, Q.Q.C.; MONTEIRO, F.A. Princípios de nutrição mineral: aspectos gerais. In: SIMPÓSIO SOBRE NUTRIÇÃO E ADUBAÇÃO DE HORTALIÇAS, 1990, Jaboticabal. Anais... Piracicaba: POTAFOS, 1993. p. 51-73. HAO, X.; PAPADOPOULOS, A.P.; DORAIS, M,; EHRET, DE; TURCOTTE, G.; GOSSELIN, A. Improving tomato fruit quality by raising the EC of NFT nutrient solutions and calcium spraying: effects on growth, photosynthesis, yield and quality. Acta Horticulturae, Leuven, v. 511, p. 213-224, 2000.

HOLCMAN, E. Microclima e produção de tomate tipo cereja em ambientes protegidos com diferentes coberturas plásticas. 2009. 127 p. Dissertação (Mestrado em Produção Vegetal)-Escola Superior de Agricultura Luiz de Queiroz, Universidade de São Paulo, Piracicaba, 2003.

KALUNGU, J. W. Resposta do tomateiro a diferentes lâminas de irrigação, doses de potássio, e cobertura do solo em ambiente protegido. 2008. 80 p. Dissertação (Mestrado em Agronomia)-Escola Superior de Agricultura Luiz de Queiroz, Universidade de São Paulo, Piracicaba, 2008.

LIMA, J. A.; DEFELIPO, B.V.; NOVAIS, R.F.; THIÉBAUT, J. T. L. Efeito das relações $\mathrm{Ca} / \mathrm{Mg}$ e $(\mathrm{Ca}+\mathrm{Mg}) / \mathrm{K}$ na correção da acidez de dois latossolos e na produção de matéria seca do tomateiro (Lycopersicum esculentum, Mill) cv. 'Kada'. Revista Ceres, v.18, p. 103-115, 1981. 
MALAVOLTA, E.; VITTI, C. G.; OLIVEIRA, A . S. Avaliação do estado nutricional das plantas: princípios e aplicações 2. ed. Piracicaba: Associação Brasileira para Pesquisa da Potassa e do Fosfato, 1997. 319 p.

MARSCHNER, H. Mineral nutrition of higher plants. 2nd ed. London: Academic Press, 1995. $889 \mathrm{p}$.

MENGEL, K. Potassium. In: BARKER, A. V.; PILBEAM, D. J. (Ed.). Handbook of plant nutrition. New York: Taylor \& Francis, 2007. 613 p.

PAIVA, E. A. S.; SAMPAIO, R. A.; MARTINEZ, H. E. P. Composition and quality of tomato fruit cultivated in nutrient solutions containing different calcium concentrations. Journal of Plant Nutrition, Philadelphia, v. 21, p. 2653-2661, 1998.

SOARES, J. M.; FARIA, M. B. Métodos de irrigação e adubação na cultura do tomate industrial. Pesquisa Agropecuária, Brasília, DF, v. 18, p. 281-286, 1983.

SOARES, I.; SOUZA, V.S. da; CRISÓSTOMO, L.A.; SILVA, L.A. da. Efeito do volume de solução nutritiva na produção e nutrição do tomateiro tipo cereja cultivado em substrato.

Revista Ciência Agronômica, Fortaleza, v. 36, n. 2, p. 152-157, maio/ago. 2005.

STANGHELLINI, C.; VAN MEURS, W.T.M.; CORVER, F.; VAN DULLEMEN, E.; SIMONSE, L. Combined effect of climate and concentration of the nutrient solution on a greenhouse tomato crop. II: yield quantity and quality. Acta Horticulturae, Wageningen, v. 458, p. 231-237, 1998. 